\title{
NON-POINTED EXACTNESS, RADICALS, CLOSURE OPERATORS
}

\author{
M. GRANDIS, G. JANELIDZE and L. MÁRKI ${ }^{凶}$ \\ (Received 11 January 2013; accepted 6 March 2013; first published online 7 June 2013)
}

\author{
Communicated by B. Gardner
}

Dedicated to Richárd Wiegandt on his 80th birthday

\begin{abstract}
In this paper it is shown how nonpointed exactness provides a framework which allows a simple categorical treatment of the basics of Kurosh-Amitsur radical theory in the nonpointed case. This is made possible by a new approach to semi-exactness, in the sense of the first author, using adjoint functors. This framework also reveals how categorical closure operators arise as radical theories.
\end{abstract}

2010 Mathematics subject classification: primary 18A40; secondary 18A20, 18A32, 18A99, 16N80, 06A15.

Keywords and phrases: nonpointed exactness, null morphisms, adjoint functors, Kurosh-Amitsur radicals, categorical closure operators.

\section{Introduction}

Classically, every ring $A$ admits short exact sequences of the form

$$
0 \rightarrow R(A) \rightarrow A \rightarrow S(A) \rightarrow 0
$$

in which $R(A)$ is the radical of $A$, and the ring $S(A)$ is semisimple; note that, here and below, rings are not required to be unital, and not even to be associative. There are several nonequivalent notions of radical, and each of them has its corresponding notion of semisimple ring. Moreover, there is a general notion, going back to old work of Kurosh and Amitsur (see, for example, [W1983] or [GW2004], and references therein), of a pair $(\mathbf{R}, \mathbf{S})$, consisting of a radical class and its corresponding semisimple class, so that:

Partially supported by: a research contract of the University of Genova, Italy, the South African NRF, and the Hungarian National Foundation for Scientific Research, grant no. K101515

(C) 2013 Australian Mathematical Publishing Association Inc. 1446-7887/2013 \$16.00 
- the classes $\mathbf{R}$ and $\mathbf{S}$ consist of rings, or, more generally, of objects in a fixed pointed category with kernels and cokernels, satisfying certain exactness conditions, for example, as in [MW1982];

- $\quad$ the classes $\mathbf{R}$ and $\mathbf{S}$ uniquely determine each other, and have several special properties, including the existence of the short exact sequence (1.1), for each ring or object $A$, in which $R(A)$ is the largest normal subobject of $A$ that is in $\mathbf{R}$, and $S(A)$ is the largest normal quotient of $A$ that is in $\mathbf{S}$;

- $\quad$ in particular, when the ground category is abelian, such a pair $(\mathbf{R}, \mathbf{S})$ is nothing but a torsion theory in the sense of Dickson [Dc1966].

Let us compare the following two well-known observations.

Observation 1.1. (a) A radical class of rings is a class $\mathbf{R}$ of rings such that a $\operatorname{ring} A$ belongs to $\mathbf{R}$ if and only if every nonzero quotient $B$ of $A$ has a nonzero ideal $C$ which is in $\mathbf{R}$.

(b) A semisimple class of rings is a class $\mathbf{S}$ of rings such that a ring $A$ belongs to $\mathbf{S}$ if and only if every nonzero ideal $B$ of $A$ has a nonzero quotient $C$ which is in $\mathbf{S}$.

ObSERVATIOn 1.2. Let $\mathbf{C}$ be an abelian category that admits arbitrary intersections of subobjects and arbitrary co-intersections of quotients. Then:

(a) a torsion class is a class $\mathbf{R}$ of objects of $\mathbf{C}$ such that an object $A$ in $\mathbf{C}$ belongs to $\mathbf{R}$ if and only if it has no nonzero morphism into any object $B$ that admits no nonzero morphism from any object $C$ in $\mathbf{R}$;

(b) a torsion-free class is a class $\mathbf{S}$ of objects of $\mathbf{C}$ such that an object $A$ in $\mathbf{C}$ belongs to $\mathbf{S}$ if and only if it has no nonzero morphism from any object $B$ that admits no nonzero morphism into any object $C$ in $\mathbf{S}$.

Observation 1.2 immediately tells us that torsion theories are defined via the Galois connection determined by the (nonsymmetric) orthogonality relation $\perp$, on the class of objects in $\mathbf{C}$, defined as

$$
A \perp B \Leftrightarrow \operatorname{Hom}(A, B)=0 ;
$$

indeed, we can rewrite conditions $1.2(\mathrm{a})$ and 1.2(b) as

$$
\mathbf{R}=\{A \in \mathbf{C} \mid \text { if, for all } C \in \mathbf{R}, C \perp B \text { then } A \perp B\}
$$

and

$$
\mathbf{S}=\{A \in \mathbf{C} \mid \text { if, for all } C \in \mathbf{S}, B \perp C \text { then } B \perp A\},
$$

respectively. In contrast to this, Observation 1.1 obviously uses two binary relations on the class of rings, but, on the other hand, there is a similarity, suggesting to rewrite 1.1(a) and 1.1(b) as

$$
\mathbf{R}=\left\{A \in \mathbf{C} \mid \text { if, for all } C \in \mathbf{R}, C \perp_{i} B \text { then } A \perp_{h} B\right\}
$$

and

$$
\mathbf{S}=\left\{A \in \mathbf{C} \mid \text { if, for all } C \in \mathbf{S}, B \perp_{h} C \text { then } B \perp_{i} A\right\},
$$


where now $\mathbf{C}$ is the category of rings, and $\perp_{i}, \perp_{h}$ are the binary relations on the class of all rings defined by

$$
B \perp_{i} A \Leftrightarrow(B \text { is not isomorphic to any nonzero ideal in } A)
$$

and

$$
A \perp_{h} B \Leftrightarrow(B \text { is not a nonzero homomorphic image of } A) \text {. }
$$

In the present paper, we begin (Section 2) with a new approach to nonpointed exactness studied by the first named author before. Surprisingly, to give a semiexact category (=ex1-category) in the sense of [Gr1992a, Gr1992b] is the same as to give a pair $\left(\mathbf{C}_{1}, \mathbf{C}_{0}\right)$ in which $\mathbf{C}_{1}$ is a category with certain pullbacks and pushouts and $\mathbf{C}_{0}$ is a full subcategory in $\mathbf{C}_{1}$ that is epi-reflective and mono-coreflective at the same time. The short Sections 3 and 4 are devoted to two main special cases: the 'standard' pointed case, where $\mathbf{C}_{0}$ is the trivial category, and the 'other extreme' case considered in [Gr1992a, Gr2013] (under the name of 'categories of pairs'), where $\mathbf{C}_{1}$ is determined by a class $\mathbf{M}$ of morphisms in $\mathbf{C}_{0}$ satisfying suitable conditions; here we shall briefly refer to the latter case as the $\mathbf{C}_{1}=\mathbf{M}$ case .

In Section 5, after recalling how a binary relation between two sets $\mathbf{X}$ and $\mathbf{Y}$ determines a Galois connection between their power sets, we explain in detail how we are modifying this construction (in a very simple way) to involve two binary relations between these two sets. Then Example 5.1 shows (many readers would say 'recalls') how these two constructions can be used as two equivalent approaches to torsion theories in abelian categories. After that we consider two nonabelian orthogonality relations $\perp_{\triangleleft}$ and $\perp_{\rightarrow}$ defined by

$$
B \perp_{\triangleleft} A \Leftrightarrow \quad \text { (there is no nonzero normal monomorphism } B \rightarrow A \text { ) }
$$

and

$$
A \perp_{\rightarrow} B \Leftrightarrow \text { (there is no nonzero normal epimorphism } A \rightarrow B \text { ), }
$$

respectively. Using these symbols we follow a tradition in classical algebra and radical theory (see, for example, [GW2004]), according to which $X \triangleleft Y$ means ' $X$ is an ideal of $Y$ ' or ' $X$ is a normal subobject of $Y$ ', while $X \rightarrow Y$ means ' $Y$ is a homomorphic image of $X$ ' or ' $Y$ is a normal quotient of $X$ '. Next we introduce our main definition (Definition 5.2), which we propose as a general framework for the theory of radicals, and prove (see Theorem 5.5) that this notion of radical and semisimple classes agrees with the usual one in Kurosh-Amitsur radical theory for pointed categories close to that of rings.

REMARK 1.3. While (1.5) and (1.6) are straightforward reformulations of well-known descriptions (see, for example, [GW2004]), and the very idea of using two binary relations (in fact, two arbitrary preorders) goes back to [FW1975], our formulation of Definition 5.2 of a radical-semisimple pair is new. Moreover, it is new even in the following cases, where, however, it is easily seen to be equivalent to known formulations: 
- the pointed case;

- for topological spaces, graphs, and the so-called abstract relational structures, where radical-semisimple pairs are called connectedness-disconnectedness theories (see [AW1975, FW1975, FW1982]).

Another surprising fact (Section 6) is: in the $\mathbf{C}_{1}=\mathbf{M}$ case radical theory becomes the theory of closure operators, with dense morphisms playing the role of radical objects and closed morphisms playing the role of semisimple objects. In particular, for the (dense, closed)-factorization $m=c d$ of $m \in \mathbf{M}$ with respect to a given closure operator, the corresponding short exact sequence (1.1) is

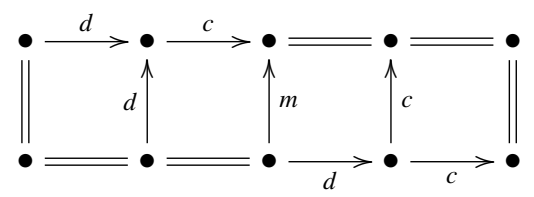

In fact it was this example that convinced us that our main definition should be given in the nonpointed context.

REMARK 1.4. (a) From the point of view of the simplicial approaches introduced in [JM2003, JM2009], it is convenient to think of the frameworks considered

- $\quad$ in this paper (that is, a set with two binary relations on it),

- $\quad$ in [JM2003],

- in [JM2009],

as one-, two-, and three-dimensional exactness structures, respectively. In subsequent papers, we plan to extend the two- and three-dimensional exactness structures to include the nonpointed context, showing how the higher-dimensional framework allows us to generalize more complex results of radical theory. Not to mention anything else, the one-dimensional exactness structure does not even allow one to formulate the existence of the short exact sequences (1.1).

(b) The 'homomorphic orthogonality' approach of (1.3) and (1.4) works also for associative rings, as shown by Gardner [G1974], if we replace all homomorphisms by homomorphisms with an accessible image, where a subring $A$ of a ring $R$ is said to be accessible if there is a finite chain $A=A_{0} \triangleleft A_{1} \triangleleft \cdots \triangleleft A_{n} \triangleleft R$. The same approach works also for 'close-to-associative' rings (like alternative rings) but not for rings in general, as noted in [JM2003] (this may have been known before).

(c) The reader might ask: what if we go back to (1.3) and (1.4), and use them in the nonpointed context to define radical-semisimple pairs? Such an approach exists and leads to interesting examples (see [AW1975, FW1975]). Also, the way Gardner looks at (pre)factorization systems in [G1994] is similar, to some extent, to the way we look at the closure operators in the above-mentioned $\mathbf{C}_{1}=\mathbf{M}$ case. 


\section{Simultaneously reflective and coreflective full subcategories versus null ideals}

Consider a diagram

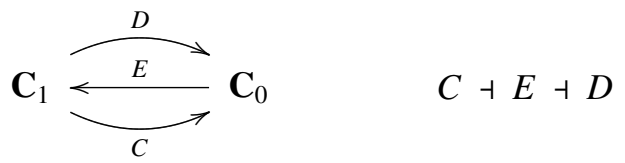

of categories and functors, in which $E$ is fully faithful and $D$ and $C$ are a right adjoint left inverse and a left adjoint left inverse of $E$, respectively. For simplicity we will assume that $\mathbf{C}_{0}$ is a full replete subcategory in $\mathbf{C}_{1}$ and $E$ is the inclusion functor, and the unit of the adjunction $E \dashv D$ and the counit of the adjunction $C \dashv E$ are identity natural transformations; the counit of $E \dashv D$ and the unit of $C \dashv E$ will be denoted by $\iota$ and $\pi$, respectively.

Since $E$ is the inclusion functor, for an object $\mathrm{A}$ in $\mathbf{C}_{1}$, we shall simply write

$$
D(A) \stackrel{\iota_{A}}{\longrightarrow} A \stackrel{\pi_{A}}{\longrightarrow} C(A) .
$$

The data (2.1) can be seen as a category, namely $\mathbf{C}_{1}$, equipped with a closed ideal $N$ of null maps in the sense of [Gr1992a, Gr1992b]. This ideal $N$ is generated by $\mathbf{C}_{0}$ in the sense that a morphism in $\mathbf{C}_{1}$ belongs to it if and only if it factors through an object in $\mathbf{C}_{0}$. At the same time $N$ determines $\mathbf{C}_{0}$ as the full subcategory of all those objects whose identity morphisms belong to it; this follows from the fact that $\mathbf{C}_{0}$ is closed under retracts.

The following table shows the precise correspondence between the terminology and notation used here and those used in [Gr1992a, Gr1992b] and subsequent papers of the same author, or in the book [Gr2013].

\begin{tabular}{|c|c|}
\hline Present paper & [Gr1992a, Gr1992b] \\
\hline \multirow[t]{2}{*}{ The data (2.1) } & $(\mathbf{X}, N)$ \\
\hline & (a category with a closed ideal) \\
\hline $\mathbf{C}_{1}$ & $\mathbf{X}$ \\
\hline $\mathbf{C}_{0}$ & $\begin{array}{l}\text { The class of null objects in } \mathbf{X} \\
\text { (objects whose identity is in } N \text { ) }\end{array}$ \\
\hline$\iota_{A}: D(A) \rightarrow A$ & $0_{A}: A_{0} \rightarrow A$ \\
\hline$\pi_{A}: A \rightarrow C(A)$ & $0^{A}: A \rightarrow A^{0}$ \\
\hline $\begin{array}{l}\text { Null morphisms (those that } \\
\text { factor through an object of } \mathbf{C}_{0} \text { ) }\end{array}$ & $\begin{array}{l}\text { Null morphisms } \\
\text { (the elements of } N \text { ) }\end{array}$ \\
\hline
\end{tabular}

Note that although the data (2.1) are stronger than a category equipped with a closed ideal, there are natural additional conditions on both of them making the resulting structures equivalent (see Theorem 2.4 below). 
As defined in [Gr1992a, Gr1992b], a semiexact category (or exl-category) is a category equipped with a closed ideal, where every morphism has a kernel and a cokernel with respect to the given ideal; such kernels and cokernels with respect to an ideal of maps had also been considered earlier by various authors (see, for example, [E1964]). We recall the definition of kernel in terms of the data (2.1) as given below.

Definition 2.1. A kernel of a morphism $f: A \rightarrow B$ in $\mathbf{C}_{1}$ (with respect to the data (2.1)) is a pair $(K, \kappa)$, where $\kappa: K \rightarrow A$ is a morphism in $\mathbf{C}_{1}$ satisfying the following conditions:

(a) $\quad f_{K}$ is a null morphism, that is, it factors through an object of $\mathbf{C}_{0}$;

(b) if $\kappa^{\prime}: K^{\prime} \rightarrow A$ is any morphism for which $f \kappa^{\prime}$ is a null morphism, then there exists a unique morphism $u: K^{\prime} \rightarrow K$ with $\kappa u=\kappa^{\prime}$.

Cokernels with respect to the data (2.1) are defined dually.

It is then natural to ask: how are these 'relative kernels' related to the usual (finite) limits in $\mathbf{C}_{1}$ ? Our answer consists of the following two easy propositions.

Proposition 2.2. The following conditions on an object $A$ in $\mathbf{C}_{1}$ are equivalent:

(a) the identity morphism $1_{A}: A \rightarrow A$ has a kernel;

(b) $\left(D(A), \iota_{A}\right)$ is a kernel of $1_{A}: A \rightarrow A$;

(c) $\iota_{A}: D(A) \rightarrow A$ is a monomorphism.

Proof. (a) $\Rightarrow($ b). Let $\mathbf{K}$ be the category of the pairs $(X, \varphi)$ in which $\varphi: X \rightarrow A$ is a null morphism in $\mathbf{C}_{1}$, and let $(K, \kappa)$ be a kernel of $1_{A}$. Then there are morphisms $u:\left(D(A), \iota_{A}\right) \rightarrow(K, \kappa)$ and $v:(K, \kappa) \rightarrow\left(D(A), \iota_{A}\right)$ in $\mathbf{K}$, and the composites $u v$ and $v u$ are identities by the universal properties of $(K, \kappa)$ and $\left(D(A), \iota_{A}\right)$, respectively.

The implication (b) $\Rightarrow$ (a) is trivial.

(b) $\Rightarrow(c)$ : Use the same straightforward argument as for ordinary kernels.

(c) $\Rightarrow$ (b): $1_{A} \iota_{A}=\iota_{A}$ is obviously a null morphism. If $\varphi=1_{A} \varphi: X \rightarrow A$ is a null morphism, then $\varphi$ is a composite of some $f: X \rightarrow Y$ and $g: Y \rightarrow A$ with $Y$ in $\mathbf{C}_{0}$. And since $Y$ is in $\mathbf{C}_{0}, g=\iota_{A} h$ for some $h$ by the universal property of $\left(D(A), \iota_{A}\right)$. Therefore, $\varphi$ factors through $\iota_{A}$. And since $\iota_{A}$ is a monomorphism, such a factorization is unique, as desired.

Proposition 2.3. Let $f: A \rightarrow B$ be a morphism in $\mathbf{C}_{1}$ for which $\iota_{B}$ is a monomorphism. Then the following conditions on a morphism $\kappa: K \rightarrow A$ are equivalent:

(a) $(K, \kappa)$ is a kernel of $f: A \rightarrow B$; 
(b) there exists a morphism $g: K \rightarrow D(B)$ making the diagram

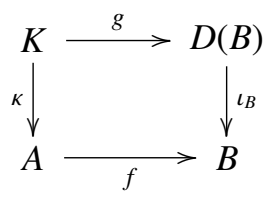

a pullback;

(c) there exists a unique morphism $g: K \rightarrow D(B)$ making the diagram (2.2) a pullback.

Proof. (a) $\Leftrightarrow\left(\right.$ b): The universal property of $\left(D(B), \iota_{B}\right)$ tells us that a morphism into $B$ is a null morphism if and only if it factors through $\iota_{B}$. After that, comparing the universal properties of a kernel and of a pullback, we see that all we need to prove is the following assertion.

(*) If $g$ makes (2.2) commute and $u: X \rightarrow K$ and $v: X \rightarrow D(B)$ are morphisms in $\mathbf{C}_{1}$ with $f \kappa u=\iota_{B} v$, then $g u=v$.

However, (*) immediately follows from the commutativity of (2.2) and the fact that $\iota_{B}$ is a monomorphism.

The equivalence of (b) and (c) follows trivially from the fact that $\iota_{B}$ is a monomorphism.

Cokernels are of course defined and constructed dually, and an easy comparison of the definitions gives the following theorem.

THEOREM 2.4. In the notation above, the following conditions are equivalent:

(a) the data (2.1) determines a semiexact category (=exl-category) in the sense of [Gr1992a], that is, every morphism in $\mathbf{C}_{1}$ has a kernel and a cokernel;

(b) for every object $A$ in $\mathbf{C}_{1}$, the morphism $\iota_{A}: D(A) \rightarrow A$ is a monomorphism that admits pullbacks along arbitrary morphisms into $A$, and the morphism $\pi_{A}: A \rightarrow$ $C(A)$ is an epimorphism that admits pushouts along arbitrary morphisms from A.

REMARK 2.5. As easily follows from the results of [Gr1992a], it is also true that every semiexact category in the sense of [Gr1992a] can be obtained as above from data (2.1) satisfying 2.4(b).

\section{Example 1: the pointed case}

This section consists of the obvious observation that when $\mathbf{C}$ is a pointed category having an initial (= terminal = zero) object, we can construct the data (2.1) by taking: 
- $\mathbf{C}_{1}=\mathbf{C}$;

- $\quad \mathbf{C}_{0}=1$, the terminal object in the category of all categories, that is, the category with exactly one morphism - although we will identify $\mathbf{C}_{0}$ with the full subcategory of all zero objects in $\mathbf{C}$.

For a given $\mathbf{C}$ this determines the data (2.1) uniquely (up to isomorphism), kernels and cokernels become the ordinary ones, and therefore semiexactness becomes equivalent to the existence of ordinary kernels and cokernels.

\section{Example 2: semiexact categories of morphisms}

In this section we choose the data (2.1) as follows. Starting with an arbitrary category $\mathbf{C}$ and a class $\mathbf{M}$ of morphisms in $\mathbf{C}$ that contains all identity morphisms, we take:

- $\mathbf{C}_{0}=\mathbf{C}$;

- $\quad \mathbf{C}_{1}$ to be the full subcategory of the arrows of $\mathbf{C}$ with objects all the morphisms in $\mathbf{M}$; the objects of $\mathbf{C}_{1}$ will be written as triples $\left(A, A^{\prime}, a\right)$, where $a: A^{\prime} \rightarrow A$ is a morphism in $\mathbf{C}$ that belongs to $\mathbf{M}$; a morphism $\left(A, A^{\prime}, a\right) \rightarrow\left(B, B^{\prime}, b\right)$ in $\mathbf{C}_{1}$ is a pair $\left(f, f^{\prime}\right)$, where $f: A \rightarrow B$ and $f^{\prime}: A^{\prime} \rightarrow B^{\prime}$ are morphisms in $\mathbf{C}$ with $f a=b f^{\prime}$;

- $\quad E: \mathbf{C}_{0} \rightarrow \mathbf{C}_{1}$ to be the diagonal functor defined by $E(A)=\left(A, A, 1_{A}\right)$, although we will identify $\mathbf{C}_{0}=\mathbf{C}$ with its replete image in $\mathbf{C}_{1}$, that is, with the category of all isomorphisms that are in $\mathbf{M}$;

- $\quad$ the functors $C$ and $D$ defined accordingly, yielding

$$
\begin{gathered}
D\left(A, A^{\prime}, a\right)=A^{\prime}=\left(A^{\prime}, A^{\prime}, 1_{A^{\prime}}\right), \quad \iota_{\left(A, A^{\prime}, a\right)}=\left(a, 1_{A}^{\prime}\right), \\
C\left(A, A^{\prime}, a\right)=A=\left(A, A, 1_{A}\right), \quad \pi_{\left(A, A^{\prime}, a\right)}=\left(1_{A}, a\right) .
\end{gathered}
$$

Proposition 4.1. Suppose the class M satisfies the following condition: for every diagram in $\mathbf{C}$ of the form

$$
S \stackrel{s}{\longrightarrow} Y \underset{g}{\stackrel{f}{\longrightarrow}} Z \stackrel{t}{\longrightarrow} T
$$

with $s$ and $t$ in $\mathbf{M}$,

$$
(f s=g s \& t f=t g) \Rightarrow f=g .
$$

Then all $\iota_{\left(A, A^{\prime}, a\right)}$ are monomorphisms and all $\pi_{\left(A, A^{\prime}, a\right)}$ are epimorphisms. In particular, this is the case if either every morphism from $\mathbf{M}$ is a monomorphism or every morphism from $\mathbf{M}$ is an epimorphism.

Proof. This is straightforward. Given morphisms

$$
\left(f, f^{\prime}\right),\left(g, g^{\prime}\right):\left(X, X^{\prime}, x\right) \rightarrow\left(A^{\prime}, A^{\prime}, 1_{A^{\prime}}\right),
$$

the equality $\iota_{\left(A, A^{\prime}, a\right)}\left(f, f^{\prime}\right)=\iota_{\left(A, A^{\prime}, a\right)}\left(g, g^{\prime}\right)$ means that

$$
\text { af }=a g, \quad f^{\prime}=g^{\prime}, \quad f x=f^{\prime}, \quad g x=g^{\prime} .
$$


Therefore, we have $a f=a g, f^{\prime}=g^{\prime}, f x=g x$, and then $\left(f, f^{\prime}\right)=\left(g, g^{\prime}\right)$ by (4.1). This proves that $\iota_{\left(A, A^{\prime}, a\right)}$ is a monomorphism. Since the required conditions are self-dual, we also conclude that $\pi_{\left(A, A^{\prime}, a\right)}$ is an epimorphism.

From this and Theorem 2.4, we obtain the following corollary.

COROLlaRy 4.2. If either every morphism from $\mathbf{M}$ is a monomorphism or every morphism from $\mathbf{M}$ is an epimorphism, and $\mathbf{C}_{1}$ admits all pullbacks along morphisms of the form $\iota_{\left(A, A^{\prime}, a\right)}$ and all pushouts along morphisms of the form $\pi_{\left(A, A^{\prime}, a\right)}$, then the data above determines a semiexact category in the sense of [Gr1992a].

Remark 4.3. Corollary 4.2 certainly applies when $\mathbf{M}$ is either a 'mono-part' or an 'epipart' of a proper factorization system in a category with pullbacks and pushouts. Both the mono case and the epi case are considered in [Gr1992a] and subsequent papers in detail.

\section{Radicals in one-dimensional exactness structures}

Let $\mathbf{X}$ and $\mathbf{Y}$ be arbitrary sets (or classes). Given a relation $\alpha: \mathbf{X} \rightarrow \mathbf{Y}$ (that is, $\alpha \subseteq \mathbf{X} \times \mathbf{Y}$ in set-theoretic notation), the corresponding Galois connection

$$
P(\mathbf{X}) \underset{\alpha^{*}}{\stackrel{\alpha_{*}}{\gtrless}} P(\mathbf{Y})
$$

between the power sets of $\mathbf{X}$ and of $\mathbf{Y}$ has $\alpha_{*}$ and $\alpha^{*}$ defined by

$$
\begin{aligned}
& \alpha_{*}(\mathbf{U})=\{Y \in \mathbf{Y} \mid \text { if } U \in \mathbf{U} \text { then } U \alpha Y\}, \\
& \alpha^{*}(V)=\{X \in \mathbf{X} \mid \text { if } V \in \mathbf{V} \text { then } X \alpha V\} .
\end{aligned}
$$

Recall also that

$$
\begin{aligned}
& \alpha_{*}(P(\mathbf{X}))=\left\{\mathbf{V} \in P(\mathbf{Y}) \mid \alpha_{*} \alpha^{*}(\mathbf{V})=\mathbf{V}\right\}, \\
& \alpha^{*}(P(\mathbf{Y}))=\left\{\mathbf{U} \in P(\mathbf{X}) \mid \alpha^{*} \alpha_{*}(\mathbf{U})=\mathbf{U}\right\},
\end{aligned}
$$

and so the maps (5.1) induce bijections

$$
\alpha^{*}(P(\mathbf{Y})) \gtrless \alpha_{*}(P(\mathbf{X}))
$$

inverse to each other.

For our purposes we need to modify the familiar data (5.1) as

$$
P(\mathbf{X}) \underset{\beta^{*}}{\stackrel{\alpha_{*}}{\rightleftarrows}} P(\mathbf{Y})
$$

where $\beta$ is another relation between the same sets. In this more general situation we have no counterpart of (5.2) of course, but we still have induced bijections

$$
\left\{\mathbf{U} \in P(\mathbf{X}) \mid \beta^{*} \alpha_{*}(\mathbf{U})=\mathbf{U}\right\} \rightleftarrows\left\{\mathbf{V} \in P(\mathbf{Y}) \mid \alpha_{*} \beta^{*}(\mathbf{V})=\mathbf{V}\right\}
$$

inverse to each other, which become the bijections (5.3) if $\alpha=\beta$. Moreover, (5.5) is the largest bijection induced by (5.4). 
In what follows we shall always have $\mathbf{X}=\mathbf{Y}$, and we will say that (5.5) is the canonical bijection between the left-closed and the right-closed subsets (with respect to $\alpha$ and $\beta$ ) in $\mathbf{X}$.

ExAmple 5.1. Let $\mathbf{C}$ be an abelian category that admits arbitrary intersections of subobjects and arbitrary co-intersections of quotients, and $\mathbf{X}$ be the class of objects in C. Then:

(a) if we take $\alpha=\beta$ to be the orthogonality relation $\perp$ defined by (1.2), then the bijections (5.3), or equivalently (5.5), are exactly the usual bijections, inverse to each other, between the torsion classes and the torsion-free classes in $\mathbf{C}$. In particular, a class of objects in $\mathbf{C}$ is left-closed if and only if it is a torsion class; similarly, a class of objects in $\mathbf{C}$ is right-closed if and only if it is a torsion-free class (see Observation 1.2 and the sentence containing (1.2)-(1.4));

(b) let us now make $\alpha$ and $\beta$ smaller and different from each other as follows:

$$
\begin{gathered}
\alpha=\{(X, Y) \in \mathbf{X} \times \mathbf{X} \mid \text { there is no nonzero mono } X \rightarrow Y\}, \\
\beta=\{(X, Y) \in \mathbf{X} \times \mathbf{X} \mid \text { there is no nonzero epi } X \rightarrow Y\} .
\end{gathered}
$$

Remarkably, it is easy to see (and known, in a sense) that although this modification yields different maps $\alpha_{*}$ and $\beta^{*}$ and different images $\alpha_{*}(P(\mathbf{X}))$ and $\beta^{*}(P(\mathbf{X}))$, it gives nevertheless the same left-closed and right-closed subsets and the same bijection between them.

There are several nonequivalent ways to generalize the data considered in this example to the context where $\mathbf{C}$ is only required to be pointed and to have kernels and cokernels. We start from 5.1(b), not 5.1(a), and generalize it as follows. As already mentioned in the Introduction, we write:

$$
\begin{gathered}
X \triangleleft Y \text { when } X \text { is (can be presented as) a normal subobject of } Y, \\
X \rightarrow Y \text { when } Y \text { is (can be presented as) a normal quotient of } X,
\end{gathered}
$$

and define the corresponding orthogonality relations $\perp_{\triangleleft}$ and $\perp_{\rightarrow}$ accordingly, that is, by (1.7) and (1.8), respectively. Since in an abelian category all monomorphisms and all epimorphisms are normal, we can say that the orthogonality relations $\perp_{\triangleleft}$ and $\perp_{\rightarrow}$ are nonabelian versions of the relations (5.6) and (5.7), respectively. Having in mind the connection with the radical theory of rings briefly described in the Introduction, we call the resulting left-closed and right-closed classes of objects radical and semisimple classes, respectively.

Let us, however, make a further generalization immediately-to the context of a semiexact category in the sense of the first author, that is, using data (2.1) satisfying the equivalent conditions of Theorem 2.4.

Definition 5.2. (a) A one-dimensional exactness structure is a system $\mathbf{X}=$ $(\mathbf{X}, \mathbf{Z}, \triangleleft, \rightarrow$ ), in which $\mathbf{X}$ is a set, $\mathbf{Z}$ is a subset in $\mathbf{X}$, and $\triangleleft$ and $\rightarrow$ are binary 
relations on $\mathbf{X}$ such that, for every $X$ in $\mathbf{X}$, there exist $Z$ and $Z^{\prime}$ in $\mathbf{Z}$ with $Z \triangleleft X$ and $X \rightarrow Z^{\prime}$.

(b) Let $\mathbf{X}=(\mathbf{X}, \mathbf{Z}, \triangleleft, \rightarrow)$ be a one-dimensional exactness structure. A pair $(\mathbf{R}, \mathbf{S})$ of subsets of $\mathbf{X}$ is said to be a radical-semisimple pair if $\mathbf{R}$ and $\mathbf{S}$ correspond to each other under the canonical bijection (5.5) for $\mathbf{Y}=\mathbf{X}$ and

$$
\begin{aligned}
& \alpha=\{(X, Y) \mid X \triangleleft Y \Rightarrow X \in \mathbf{Z}\}, \\
& \beta=\{(X, Y) \mid X \rightarrow Y \Rightarrow Y \in \mathbf{Z}\} .
\end{aligned}
$$

That is, $(\mathbf{R}, \mathbf{S})$ is a radical-semisimple pair if and only if $\mathbf{R}=\beta^{*}(\mathbf{S})$ and $\mathbf{S}=\alpha_{*}(\mathbf{R})$. Accordingly, the radical (semisimple) classes with respect to $\mathbf{X}$ correspond to the leftclosed (right-closed) classes with respect to relations (5.8), (5.9) in the pointed case (when $\mathbf{Z}$ is the set of zero objects).

(c) For data (2.1) satisfying the equivalent conditions of Theorem 2.4, a pair $(\mathbf{R}, \mathbf{S})$ of classes of objects of $\mathbf{C}_{1}$ is said to be a radical-semisimple pair if it in the sense of (b), where:

- $\quad \mathbf{X}$ is the class of objects in $\mathbf{C}_{1}$;

- $\quad \mathbf{Z}$ is the class of objects in $\mathbf{C}_{0}$;

- $X \triangleleft Y$ when $X$ is a normal subobject of $Y$, that is, there exist morphisms $\kappa: X \rightarrow$ $Y$ and $f: Y \rightarrow Y^{\prime}$ such that $\kappa$ is the kernel of $f$ (in the sense of Definition 2.1);

- $\quad X \rightarrow Y$ when $Y$ is a normal quotient of $X$, that is, there exist morphisms $g: X^{\prime} \rightarrow$ $X$ and $\lambda: X \rightarrow Y$ such that $\lambda$ is the cokernel of $g$ (in the sense of Definition 2.1). A class $\mathbf{Y} \subseteq \mathbf{X}$ is said to be a radical class (semisimple class) if $\mathbf{Y}=\beta^{*} \alpha_{*}(\mathbf{Y})(\mathbf{Y}=$ $\left.\alpha_{*} \beta^{*}(\mathbf{Y})\right)$.

The following two propositions are in fact nothing but explicit reformulations of Definition 5.2(b).

Proposition 5.3. Let $\mathbf{X}=(\mathbf{X}, \mathbf{Z}, \triangleleft, \rightarrow)$ be a one-dimensional exactness structure. $A$ subset $\mathbf{R}$ in $\mathbf{X}$ is a radical class if and only if it satisfies the following conditions:

(a) if $A$ is in $\mathbf{R}$, then, for every $B \in \mathbf{X} \backslash \mathbf{Z}$ with $A \rightarrow B$, there exists $C \in \mathbf{R} \backslash \mathbf{Z}$ with $C \triangleleft B$;

(b) given $A$ in $\mathbf{X}$, if, for every $B \in \mathbf{X} \backslash \mathbf{Z}$ with $A \rightarrow B$, there exists $C \in \mathbf{R} \backslash \mathbf{Z}$ with $C \triangleleft B$, then $A$ is in $\mathbf{R}$.

Proof. Just observe that conditions (a) and (b) are equivalent to the inclusions $\mathbf{R} \subseteq$ $\beta^{*} \alpha_{*}(\mathbf{R})$ and $\beta^{*} \alpha_{*}(\mathbf{R}) \subseteq \mathbf{R}$, respectively.

Proposition 5.4. Let $\mathbf{X}=(\mathbf{X}, \mathbf{Z}, \triangleleft, \rightarrow)$ be a one-dimensional exactness structure. $A$ subset $\mathbf{S}$ in $\mathbf{X}$ is a semisimple class if and only if it satisfies the following conditions:

(a) if $A$ is in $\mathbf{S}$, then, for every $B \in \mathbf{X} \backslash \mathbf{Z}$ with $B \triangleleft A$, there exists $C \in \mathbf{S} \backslash \mathbf{Z}$ with $B \rightarrow C$;

(b) given $A$ in $\mathbf{X}$, if, for every $B \in \mathbf{X} \backslash \mathbf{Z}$ with $B \triangleleft A$, there exists $C \in \mathbf{S} \backslash \mathbf{Z}$ with $B \rightarrow C$, then $A$ is in $\mathbf{S}$. 
Proof. Just observe that conditions (a) and (b) are equivalent to the inclusions $\mathbf{S} \subseteq$ $\alpha_{*} \beta^{*}(\mathbf{S})$ and $\alpha_{*} \beta^{*}(\mathbf{S}) \subseteq \mathbf{S}$, respectively.

Conditions 5.3(a), 5.3(b), 5.4(a), 5.4(b) of these propositions are straightforward generalizations of the standard radical-theoretic conditions (R1), (R2), (S1), (S2), see, for example, [W1983] or [GW2004]. This proves the following theorem.

THeOREM 5.5. Let the data (2.1) be chosen as in Section 3, and let $\mathbf{C}$ be the category of rings, or, more generally, any category satisfying one of the essentially equivalent sets of axioms in [MW1982] (see Remark 5.6(e) below).

Then the radical and the semisimple classes (with respect to these data) in the sense of Definition 5.2(c) are the same as the radical and the semisimple classes in the sense of the usual Kurosh-Amitsur radical theory. Furthermore, the radical-semisimple pairs are the same as the pairs consisting of a radical class and a semisimple class corresponding to each other in the usual sense.

REMARK 5.6. (a) Let us reconsider the passage from torsion theories to radicals. It consists of the following steps.

- We recall (see Example 5.1(a)) that torsion theories in an abelian category $\mathbf{C}$ are defined via the Galois connection determined by the orthogonality relation $\perp$ defined by (1.2).

- $\quad$ Next, we observe (see Example 5.1(b)) that the same can be done in a slightly different way by using two (smaller) orthogonality relations that use mono- and epimorphisms instead of all morphisms.

- When $\mathbf{C}$ is not abelian, we choose those two orthogonality relations to be $\perp_{\triangleleft}$ and $\perp_{\rightarrow}$ defined by (1.7) and (1.8), respectively. And the accordingly modified notions of torsion and torsion-free classes give us the notions of radical and semisimple classes, respectively. Since these $\perp_{\triangleleft}$ and $\perp_{\rightarrow}$ are defined using normal mono- and epimorphisms in the same way as we used mono- and epimorphisms before, this does not change anything in the abelian case.

(b) Why not use a single orthogonality relation in nonabelian cases? The answer is: that approach would not be suitable for the Kurosh-Amitsur radical theory in general (see Remark 1.4(b)).

(c) Since the structure involving two orthogonality relations is relatively complicated, many explicit formulations of 'obvious' radical-theoretic results are useful. For instance, let us mention that $(\mathbf{R}, \mathbf{S})$ is a radical-semisimple pair in $\mathbf{X}$ if and only if

$$
\begin{gathered}
\mathbf{R}=\{X \in \mathbf{X} \mid \text { every } S \in \mathbf{S} \text { with } X \rightarrow S \text { is in } \mathbf{Z}\}, \\
\mathbf{S}=\{X \in \mathbf{X} \mid \text { every } R \in \mathbf{R} \text { with } R \triangleleft X \text { is in } \mathbf{Z}\} .
\end{gathered}
$$

Note also that the above-mentioned conditions (R1), (R2), (S1), (S2), and so also Propositions 5.3 and 5.4, are essentially the same as Observation 1.1 (for rings, but similarly in general). 
(d) As we see, for example, from Proposition 5.4, we could, alternatively, simply use the set $\mathbf{X} \backslash \mathbf{Z}$ (instead of both $\mathbf{X}$ and $\mathbf{Z}$ ), but that small simplification would become inconvenient for considering examples, and also for considering two- and three-dimensional exactness structures as we plan in subsequent papers.

(e) In the pointed case considered in Section 3, requiring further conditions on the ground category $\mathbf{C}$, one obtains various standard results of the theory of KuroshAmitsur radicals. This can be done when the category $\mathbf{C}$ satisfies any of the (essentially equivalent) systems of axioms considered in [MW1982]. As follows from the results of [MW1982], this holds, for instance, if $\mathbf{C}$ is Kelly well-complete (that is, small complete and has arbitrary intersections of subobjects) and semi-abelian in the sense of [JMT2002].

(f) An important special case of the previous item, considered by many authors, is the case of $\mathbf{C}$ being a variety of groups with multiple operators in the sense of Higgins [H1956]. Although this case is considered in [W1983], most of the material of [W1983] is devoted to the more special case of rings; for this and especially for the associative case, see [GW2004].

\section{Towards generalized closure operators}

In this section we make a simple analysis of Definition 5.2(c) in the situation considered in Section 4 under the assumptions of Corollary 4.2 and assuming for simplicity that the class $\mathbf{M}$ is the 'mono-part' of a proper factorization system in a category with pullbacks and pushouts (see Remark 4.3). As is shown in [Gr1992a] and can be easily checked directly, we have the following proposition.

Proposition 6.1. (a) A morphism $\left(f, f^{\prime}\right):\left(A, A^{\prime}, a\right) \rightarrow\left(B, B^{\prime}, b\right)$ in $\mathbf{C}_{1}$ is a normal monomorphism (that is, the kernel of some morphism in the sense of Definition 2.1) if and only if $f$ is in $\mathbf{M}$ and $f^{\prime}$ is an isomorphism.

(b) A morphism $\left(f, f^{\prime}\right):\left(A, A^{\prime}, a\right) \rightarrow\left(B, B^{\prime}, b\right)$ in $\mathbf{C}_{1}$ is a normal epimorphism (the cokernel of some morphism in the sense of Definition 2.1) if and only if $f$ is an isomorphism and $f^{\prime}$ is in $\mathbf{M}$.

Theorem 6.2. Under the assumptions above, two subclasses $\mathbf{R}$ and $\mathbf{S}$ of $\mathbf{M}$ form a radical-semisimple pair $(\mathbf{R}, \mathbf{S})$ if and only if the following conditions hold for every $m \in \mathbf{M}$ :

(a) $\quad m$ is in $\mathbf{R}$ if and only if for every $s \in \mathbf{S}$ and $n \in \mathbf{M}$ with $m=$ sn, the morphism $s$ must be an isomorphism;

(b) $m$ is in $\mathbf{S}$ if and only if for every $r \in \mathbf{R}$ and $n \in \mathbf{M}$ with $m=n r$, the morphism $s$ must be an isomorphism.

As Theorem 6.2 shows, the classes $\mathbf{R}$ and $\mathbf{S}$ have the same properties as the classes of dense and closed morphisms with respect to a closure operator, considered in various contexts, for instance, by Dikranjan and Tholen [DkT1995]. This suggests using this theorem as a definition of a generalized closure operator. 


\section{References}

[AW1975] A. V. Arkhangel'skii and R. Wiegandt, 'Connectednesses and disconnectednesses in topology', Gen. Top. Appl. 5 (1975), 9-33.

[Dc1966] S. E. Dickson, 'A torsion theory for abelian categories', Trans. Amer. Math. Soc. 121 (1966), 223-235.

[DkT1995] D. Dikranjan and W. Tholen, Categorical Structure of Closure Operators (Kluwer Academic Publications, Dordrecht, 1995).

[E1964] C. Ehresmann, 'Cohomologie à valeurs dans une catégorie dominée, Extraits du Colloque de Topologie, Bruxelles, 1964', in: Oeuvres Complètes et Commentées, Partie III-2 (ed. C. Ehresmann) (Amiens, 1980), 531-590.

[FW1975] E. Fried and R. Wiegandt, 'Connectednesses and disconnectednesses of graphs', Algebra Universalis 5 (1975), 411-428.

[FW1982] E. Fried and R. Wiegandt, 'Abstract relational structures, II (Torsion theory)', Algebra Universalis 15 (1982), 22-39.

[G1974] B. J. Gardner, 'Some radical constructions for associative rings', J. Aust. Math. Soc. 18 (1974), 442-446.

[G1994] B. J. Gardner, 'Morphic orthogonality and radicals in categories', in: Rings and Radicals (Proc. Conf. Shijiazhuang, 1994), Pitman Research Notes Mathematical Series, 346 (Longman, Harlow, 1996), 178-206.

[GW2004] B. J. Gardner and R. Wiegandt, Radical Theory of Rings, Pure and Applied Mathematics, 261 (Marcel Dekker, New York, 2004).

[Gr1992a] M. Grandis, 'A categorical approach to exactness in algebraic topology', in: Atti del V Convegno Nazionale di Topologia, Lecce-Otranto 1990, Rend. Circ. Mat. Palermo 29 (1992), 179-213.

[Gr1992b] M. Grandis, 'On the categorical foundations of homological and homotopical algebra', Cah. Topol. Géom. Differ. Catég. 33 (1992), 135-175.

[Gr2013] M. Grandis, Homological Algebra in Strongly Non-Abelian Settings (World Scientific, Hackensack NJ, 2013).

[H1956] P. J. Higgins, 'Groups with multiple operators', Proc. Lond. Math. Soc. (3) 6 (1956), 366-416.

[JM2003] G. Janelidze and L. Márki, 'Kurosh-Amitsur radicals via a weakened Galois connection', Comm. Algebra 31 (2003), 241-258.

[JM2009] G. Janelidze and L. Márki, 'A simplicial approach to factorization systems and KuroshAmitsur radicals', J. Pure Appl. Algebra 213 (2009), 2229-2237.

[JMT2002] G. Janelidze, L. Márki and W. Tholen, 'Semi-abelian categories', J. Pure Appl. Algebra 168 (2002), 367-386.

[MW1982] L. Márki and R. Wiegandt, 'Remarks on radicals in categories', in: Category Theory. Applications to Algebra, Logic and Topology (Proc. Conf. Gummersbach, 1981), Lecture Notes in Mathematics, 962 (Springer, 1982), 190-196.

[W1983] R. Wiegandt, 'Radical theory of rings', Math. Student 51 (1983), 145-185.

M. GRANDIS, Dipartimento di Matematica, Università di Genova,

Via Dodecaneso 35, 16146-Genova, Italy

e-mail: grandis@dima.unige.it

G. JANELIDZE, Department of Mathematics and Applied Mathematics, University of Cape Town, Rondebosch 7701, South Africa

e-mail: George.Janelidze@uct.ac.za

L. MÁRKI, Alfréd Rényi Institute of Mathematics, Hungarian Academy of Sciences, H-1364 Budapest, Pf. 127, Hungary e-mail: marki.laszlo@ renyi.mta.hu 